$\underline{\underline{\beta}}=$

\title{
Impact of raw cocoa and chocolate on lipid profile and liver function in alcohol-induced toxicity Wister rats
}

\author{
Alaa Abdel Gaffer Ahmed Saleh *, Murwan Khalid Sabahelkhier \\ Department of Biochemistry and Molecular Biology, Faculty of Science and Technology, Al-Neelain University, Khartoum, SUDAN \\ *Corresponding author E-mail:Alabbadeya.2@hot mail.com
}

\begin{abstract}
The aim of study is to evaluate the impact of raw cocoa and processed cocoa (galaxy chocolate) in the treatment of alcoholic fatty liver compare to prednisolone drug as common medication to treat fatty liver and carried out in Al-Neelain University in 2016. Eighteen female Wister rats were classified into six groups: Group one represented negative $\left(\mathrm{H}_{2} \mathrm{O}\right)$, Group two positive controls $(0.5 \mathrm{ml} / 40 \%$ alcohol), Group three represented doses of $0.67 \mathrm{~g} \mathrm{~kg}$ raw cocoa, Group four represented dose of $1.35 \mathrm{~g} / \mathrm{kg}$ raw cocoa, Group five represent dose of $5.4 \mathrm{~g} / \mathrm{kg}$ galaxy chocolate, Group six received dose of $4.8 \mathrm{mg} / \mathrm{kg}$ prednisolone. The results show that the cocoa with low and high doses, galaxy chocolate and prednisolone significantly reduce the ALT, AST and GGT, and a significant decrease in the ALT level after administration of prednisolone drug when compared high cocoa dose group. Whereas, Galaxy chocolate significantly reduce the AST compared to low dose cocoa treated group. Also there is a significant increase in HDL and decrease in Total cholesterol, Triglycerol and low density lipoprotein in all treated groups compared to positive control. The ratio between Triglycerol: HDL and LDL: HDL show a significant reduction in group treated with galaxy chocolate compared to other treated groups. The results concluded that, cocoa in both forms raw and processed are efficient in the treatment of alcoholic fatty liver in comparison with prednisolone drug.
\end{abstract}

Keywords:Galaxy Chocolate; Raw Cocoa; Prednisolone and Alcohol.

\section{Introduction}

"Alcoholic liver disease (ALD) has long been one of the most prevalent and devastating conditions caused by alcohol consumption" (Mandrekar\&Szabo 2009). It considered as a complex and multifaceted pathological process, including oxidative stress, inflammation and fatty acid overproduction (Ding et al. 2012). Alcohol intake results in serious and severe damage in liver because the action of their metabolites in metabolic reactions; the acetaldehyde cause mitochondrial and microtubule damage which reduce the lipid oxidation and accumulation of very low density lipoprotein (VLDL) (EASL 2012). Also, the alcoholtransformation lead to excessive reduction in the NAD to form NADH; this stimulate the fatty acid and Triglycerol production and ketogensis while the mitochondrial $\beta$ - oxidation and gluconeogenesis pathways are inhibited, also the ATP synthesis in mitochondria was depleted due to the effect of alcohol on cytochromes and ATP synthase that involve in ATP formation (Gramenzi et al. 2006). On other hands the free radicals and hydroxyl-ethyl radicals which are produced from the acetaldehyde; react with cellular components and induce the inflammation through activation of inflammatory cytokines like (TNF_ $\alpha$ ) (EASL 2012), and TGF- $\beta$ which involve in the fibrinogensis process and fibrosis (Gramenzi et al. 2006). "Over the past decade, herbal medicines have attracted much attention as potential therapeutic agents in the prevention and treatment of ALD, due to their multiple targets and less toxic side effects" (Ding et al. 2012). Recently; cocoa have been used in the treatment of various diseases because it possess the antioxidant poly phenols which 2-3 times higher than green tea (Subhashini et al. 2010). The polyphenols found in cocoa have several important activities such as: scavenging activity of gallic acid and epicatechin can prevent the free radicals- induced cell damage (Andujar et al. 2012), antioxidant activity of polyphenols also "up regulate antioxidant defenses involved in the cellular stress response" and increase the expression of anti-oxidants and detoxifying proteins(Scapagniniet al. 2014), flavonoids also reverse the fat accumulation and inflammation and decrease the level of 4- hydroxynonenal, a product of lipid peroxidation (Mckimet al. 2002).endothelial nitric oxide synthase (e NOS) stimulation by polyphenols make it a powerful anti-hypertensive agent(Scapagnini et al. 2014). Metabolic activation of carcinogens and the activity of vascular endothelial growth factor were inhibited by the actions of polyphenols (Andujar et al. 2012). The NCaffeoyldopamine isolated fromTheobroma cacao tree, have hepatoprotective effect due to its antioxidant activity by increasing superoxide dismutase and glutathionelevels in hepatic tissue and significantly lowering the liver-enzyme levels (AST, ALT and ALP), as well as the lipid peroxidation in liverthrough the CYP4502E1 down-regulation (Arellanes et al. 2016) ; so cocoa in both forms; raw and processed was used in medicine to treat various diseases like diabetes mellitus, cardiovascular diseases and cancer (Andujar et al. 2012).

Objective of study:

The current study conducted to evaluate the biochemical and histopathological sections of liver among groups treated with cocoa and chocolate compared to standard drug (prednisolone). 


\section{Materials and methods}

\subsection{Materials}

Raw cocoa was brought from market in Khartoum, North Sudan galaxy chocolate was obtained from Mars incorporated company USA. Prednisolone drug was obtained from pharmacy in Khartoum, North Sudan.

\subsubsection{Experimental animals}

Eighteen female Wister rats with average weight of (76-132 g) classified randomly into six groups, group one (negative control), group two (positive control), group three (cocoa low dose 0.67 $\mathrm{g} / \mathrm{kg}$ ), group four (cocoa high dose $1.35 \mathrm{~g} / \mathrm{kg}$ ), group five (galaxy chocolate $5.4 \mathrm{~g} / \mathrm{kg}$ ) and group six (prednisolone $4.8 \mathrm{mg} / \mathrm{kg}$ ), The rats were clinically healthy and housed under standard husbandry condition $\left(30 \pm 2^{\circ} \mathrm{C}, 60\right.$ to $70 \%$ relative humidity $12 \mathrm{~h}$ : $12 \mathrm{~h}$ day night cycle) and fed on rat's diet. Animal experiments were designed in accordance of institutional animal ethical committee. Group one received water as placebo for four months, while other remaining five groups were received $0.5 \mathrm{ml} / 40 \%$ ethanol alcohol for two months to induce fatty liver, then, for two months cocoa low and high doses, galaxy chocolate and prednisolone wereadministrated with doses $0.67 \mathrm{~g} / \mathrm{kg}, 1.35 \mathrm{~g} / \mathrm{kg}, 5.4 \mathrm{~g} / \mathrm{kg}$ and 4.8 $\mathrm{mg} / \mathrm{kg}$ respectively.

\subsubsection{Serobiochemical parameters}

Blood samples were collected after scarification under mild chloroform anesthesia, then serum was obtained by centrifuged at $4000 \mathrm{rpm}$ and stored at $-20^{\circ} \mathrm{C}$ till used.

\subsection{Methods}

\subsubsection{Preparation of cocoa, chocolate and prednisolone}

5 grams from cocoa powder was mixed with $140 \mathrm{ml}$ of distilled water and used as stock Solution. Finally the stock suspension was diluted to appropriate doses concentration. Galaxy chocolate was prepared by dissolving one piece (5.6 grams) of it in $20 \mathrm{ml}$ dis- tilled water and used directly. Prednisolone single tablet was dissolved in $1 \mathrm{ml}$ distilled water and become ready to use

\subsubsection{Serobiochemical methods}

Blood samples were analyzed for liver enzymes (ALT, AST and GGT) and lipid profiles (Total cholesterol, triglyceride, HDL and LDL) using spectrophotometer JANAWAY 3600.

\subsubsection{Histological methods}

Autopsy samples were taken from the hepatic tissues of the different animal groups, fixed in $4 \%$ formalin for $24 \mathrm{~h}$. The specimens were cleared in xylene embedded in paraffin in hot air oven at 56 ${ }^{\circ} \mathrm{C}$ for $24 \mathrm{~h}$. Paraffin bees wax tissue blocks were prepared for sectioning at 5-mm thickness using a rotatory microtome. The obtained tissue sections were collected on glass slides, dehydrated by immersing in serial dilutions of ethyl alcohol-water mixture, cleaned in xylene and embedded in paraffin wax. Next, the specimens were de-paraffinized and stained with hematoxylin and eosin (H\&E) dye for histopathological examinations. Photomicrographs of the tissue sections were captured and seen under light microscope at $\times 400$ magnification power (Chiedozie et al.2015).

\subsection{Statistical methods}

Analysis of variance (ANOVA) with post hoc analysis were employed to test whether the effect of cocoa, chocolate and prednisolone in the mean concentration of liver enzymes (ALT, AST and GGT) and lipid profile (TG, cholesterol, HDL and LDL) were significantly difference in study groups. Also, the data obtains were analyzed with multiple comparison test (LSD) to compare between groups. All results are presented as Mean \pm SEM, with the level of significanceat $\mathrm{P}$-value $<0.05$.

\section{Results}

\subsection{Comparison between the mean concentrations in the activity of liver enzymes (ALT and AST):}

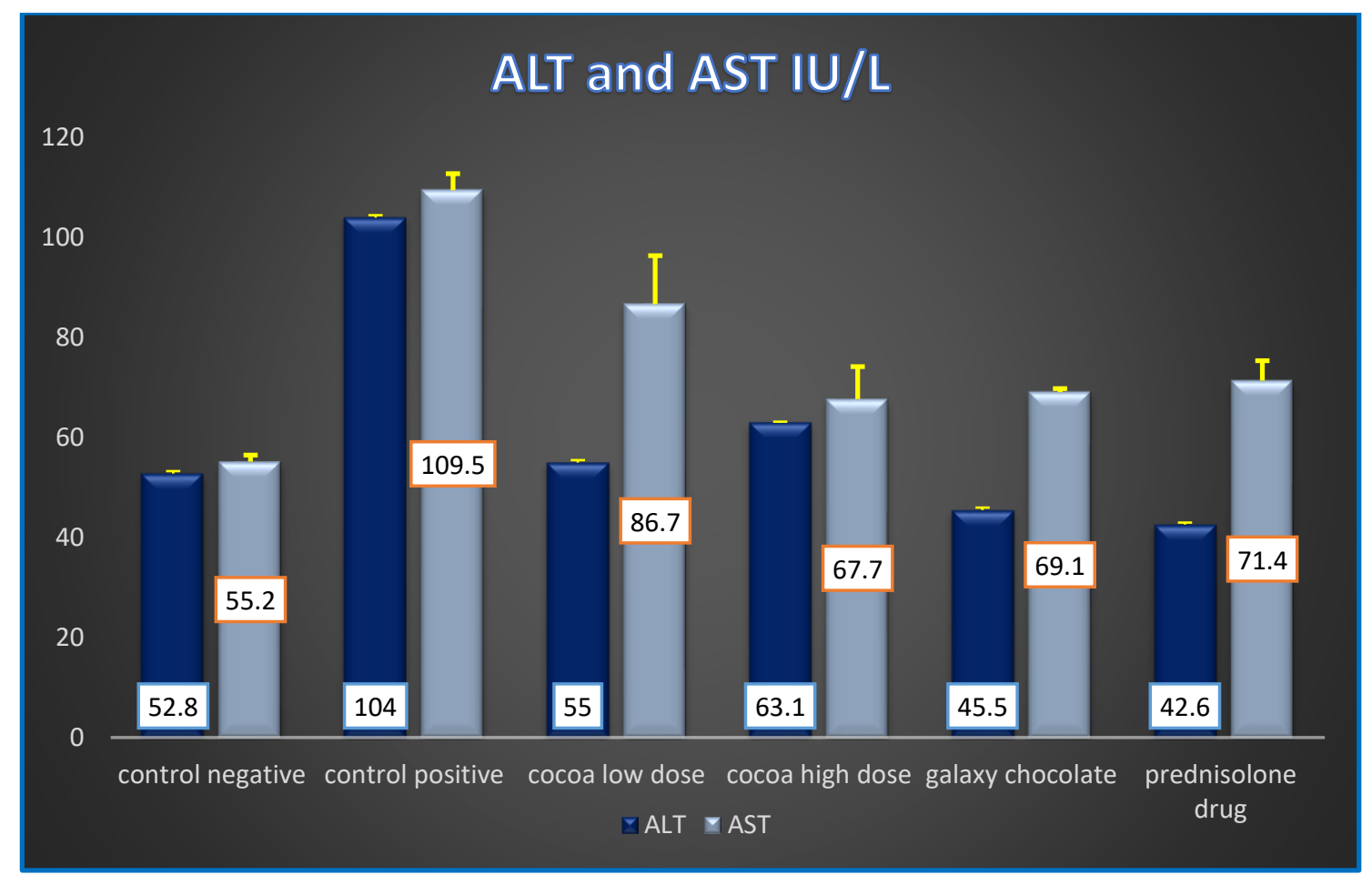

Fig. 1: Show a significant decrease in the mean concentration of plasma ALT when use cocoa ( 0.67 and $1.35 \mathrm{G} / \mathrm{Kg})$, Galaxy Chocolate and Prednisolone Compared to the positive control with $\mathrm{p}$-value $(\mathrm{P}-\mathrm{Value}=0.000)$. Also There was a significant decrease in the ALT level after administration of Prednisolone drug when compared high cocoa dose group $(\mathrm{P}=0.017)$. 
Also this figure Show a significant decrease in the mean of plasma AST concentration when use cocoa $(0.67$ and $1.35 \mathrm{~g} / \mathrm{kg})$, galaxy chocolate and prednisolone compared to positive control with $\mathrm{p}$ value ( $p$-value $=0.000$ ). Also there was a significant decrease in the AST level after administration of cocoa $(1.35 \mathrm{~g} / \mathrm{kg})$ compared to group treated by low dose of cocoa $(0.67 \mathrm{~g} / \mathrm{kg})(\mathrm{p}=0.004)$. Galaxy chocolate significantly reduce the mean concentration of AST compared to low dose cocoa treated group $(\mathrm{p}=0.006)$.

\subsection{Comparison between the mean concentrations in} the activity of GGT

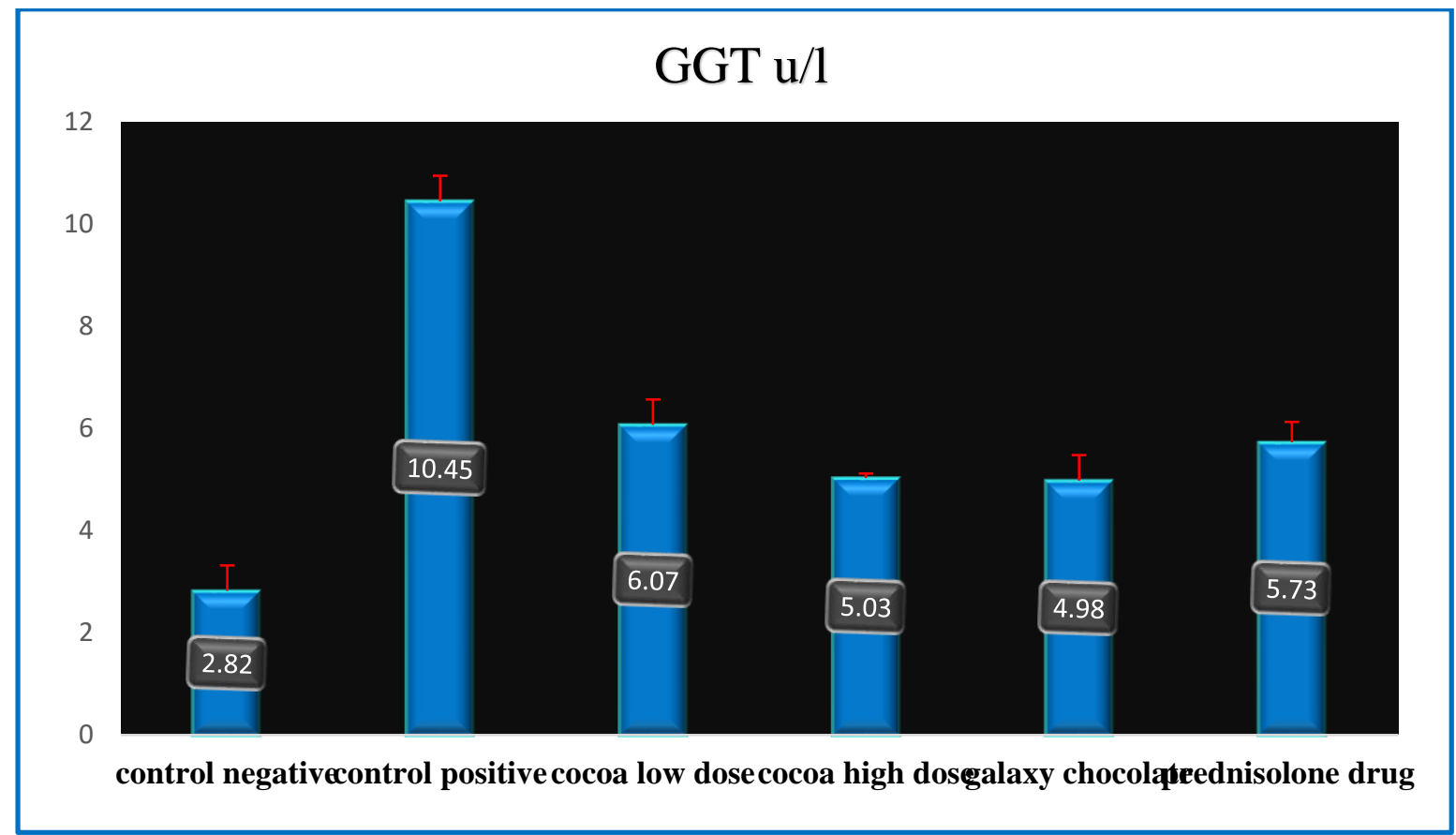

Fig. 2: Show a significant decrease in the mean concentration of plasma GGT after treatment with cocoa $(0.67$ and $1.35 \mathrm{G} / \mathrm{Kg})$, Galaxy Chocolate and Prednisolone compared to the positive control (P-Value $=0.000)$.

\subsection{Comparison the mean concentrations of total cholesterol and LDL among treated groups}

\section{Total cholesterol and LDL $\mathrm{mg} / \mathrm{dl}$}

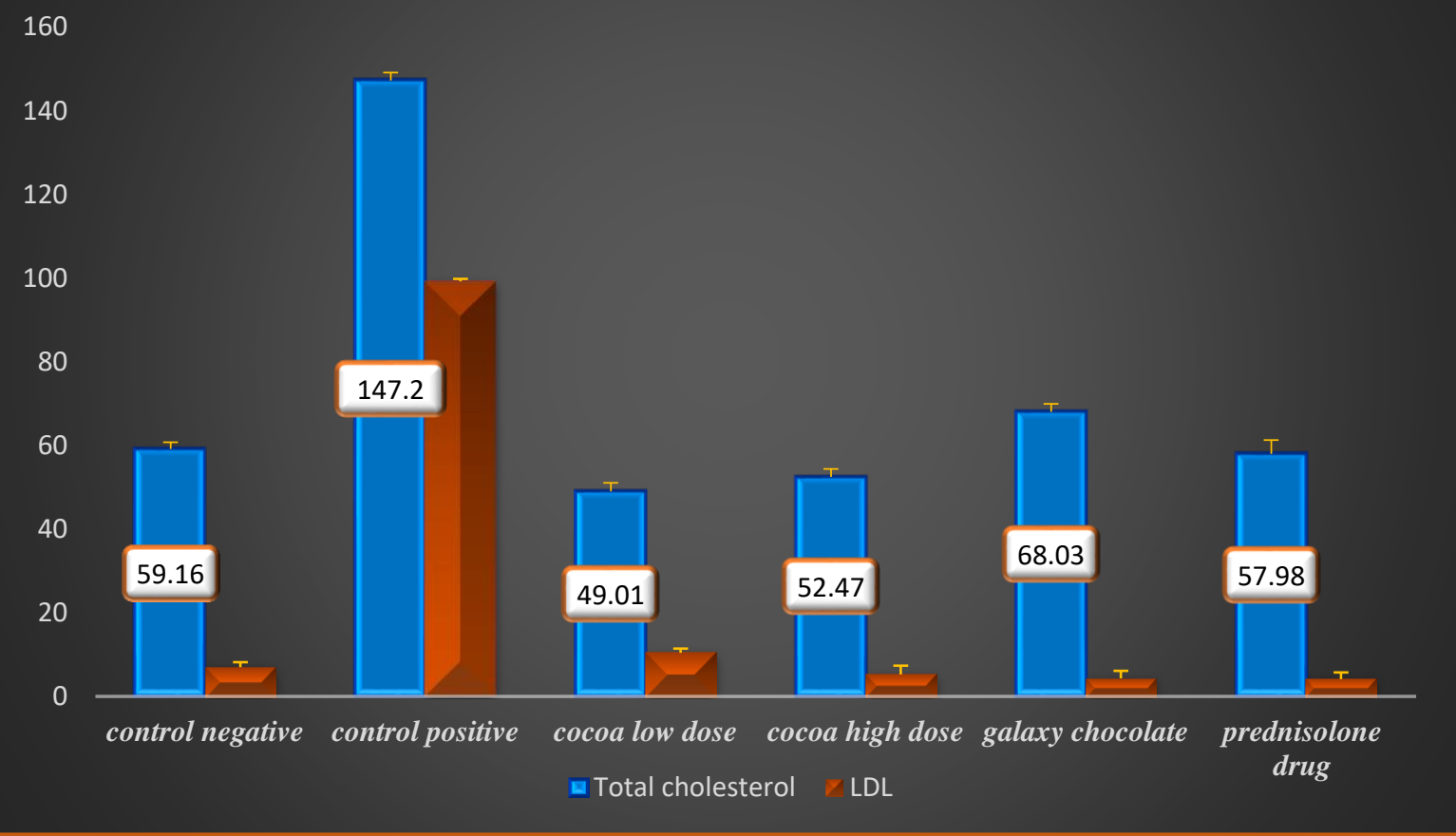

Fig. 3: Show a Significant decrease in the mean concentrations of plasma total cholesterol and LDL when use cocoa (0.67 and 1.35 G/Kg), Galaxy Chocolate and Prednisolone compared to positive control with p-value $(\mathrm{P}-\mathrm{Value}=0.000)$. Also there was significant decreased in the total cholesterol level after cocoa administration $(0.67 \mathrm{~g} / \mathrm{Kg})$ compared to Galaxy Chocolate treated group $(\mathrm{P}=0.000)$. Group administrated Prednisolone has significant reduction in LDL concentration compared to Galaxy Chocolate and low cocoa doses $(\mathrm{P}=0.007,0.013)$, respectively. High dose of cocoa significantly decreased the LDL levels compared to group received low cocoa dose $(\mathrm{P}=0.02)$. 


\subsection{Comparison the mean concentrations of triglycerol and HDL among treated groups}

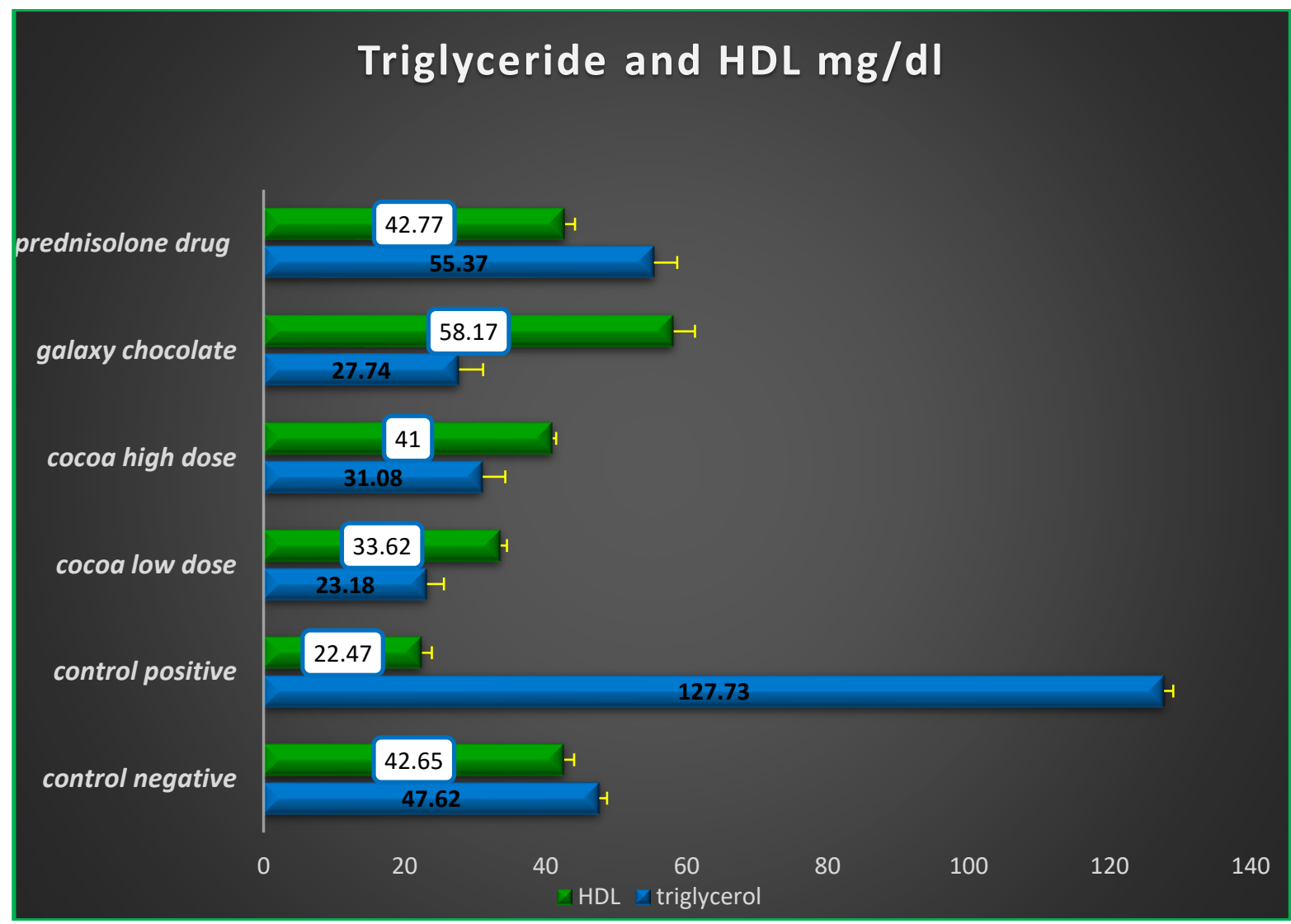

Fig. 4: Show a significant decrease in the mean concentration of plasma Triglyceride and increased in the level HDL after administration of cocoa ( 0.67 and $1.35 \mathrm{G} / \mathrm{Kg}$ ), Galaxy Chocolate and Prednisolone compared to positive control with p-value (P-Value $=0.000)$. Also there was a significant decreased in the Triglyceride level after cocoa administration $(0.67 \mathrm{~g} / \mathrm{Kg}$ and $1.35 \mathrm{~g} / \mathrm{Kg})$ and Galaxy Chocolate compared to Prednisolone treated group $(\mathrm{P}=0.000,0.000$ and 0.000 ), respectively. Also the HDL was increased significantly in Galaxy Chocolate treated group compared to that treated with low, high cocoa doses and Prednisolone drug $(\mathrm{P}=0.000,0.000$ and 0.000$)$, respectively. Prednisolone show significant increased in HDL concentration in comparison with low cocoa treated group $(\mathrm{P}=0.002)$. There was a significant differences between groups administrated low and high cocoa doses $(\mathrm{P}=0.008)$.

\subsection{Comparison the ratio between triglyceride (TG): HDL and LDL: HDL among treated groups}

Table 1: Show Ratio of TG: HDL and Ratio of LDL: HDL for Six Groups.

\begin{tabular}{lll}
\hline Groups & TG:HDL & LDL:HDL \\
\hline Negative control & 1.12 & 0.16 \\
Positive control & 5.73 & 4.45 \\
Cocoa low dose & 0.61 & 0.32 \\
Cocoa high dose & 0.76 & 0.13 \\
Galaxy chocolate & 0.476 & 0.08 \\
Prednisolone drug & 1.293 & 0.10 \\
\hline
\end{tabular}

Table 1 Show significant decrease in TG: HDL and LDL: HDL when use galaxy chocolate in comparison with Positive control $(\mathrm{P}-$ value $=0.000$ ). The TG: HDL ratio was significantly decreased in galaxy chocolate treated group compared to cocoa low dose $(0.67$ $\mathrm{g} / \mathrm{kg})(\mathrm{p}$-value $=0.02)$. Also this ratio had significant decreased in high dose cocoa $(1.35 \mathrm{~g} / \mathrm{kg})$ and galaxy chocolate compared to prednisolone treated group ( $\mathrm{p}$-value $=0.046,0.005$ ) respectively.

\subsection{Histological results}


(A)

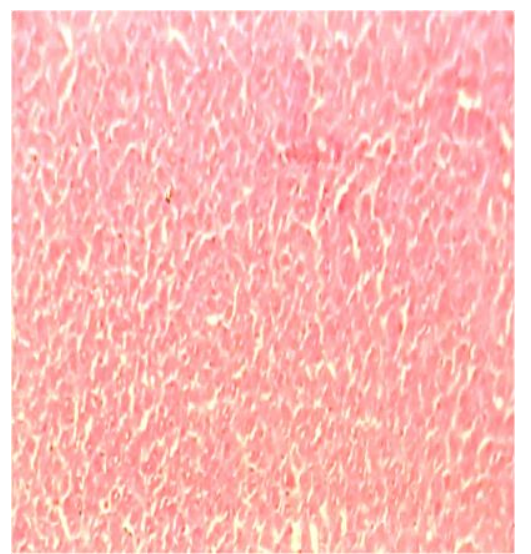

Normal liver histology in negative control (X-40).

(C)

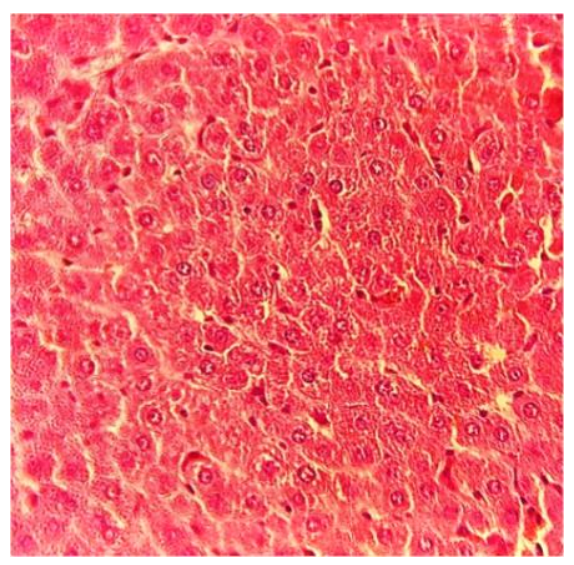

Show absence of necrosis and fat accumulation in liver cell after prednisolone administration in comparison to alcoholic group (X-40).

(E)

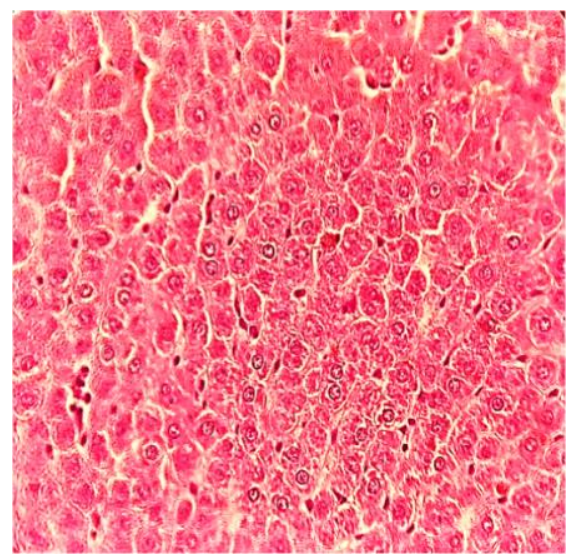

Show absence of lipid deposition and necrosis in liver cell in high dose cocoa treated group compared toAlcoholic group (X-40).

\section{Discussion}

Alcoholic liver disease represents a spectrum of clinical illness and morphological changes that range from fatty liver to hepatic inflammation and necrosis (alcoholic hepatitis) to progressive fibrosis (alcoholic cirrhosis)" ,The ALD mortality in different countries in the world was increased significantly in last decade (Gramenzi 2006). The efficient treatment for the fatty liver_ the early step in liver diseases whatever it causes; become a problem and the available medications used including corticosteroids have adverse effects, so natural treatment may be more safe and efficient than other drugs. The present study conducted to evaluate the
(B)

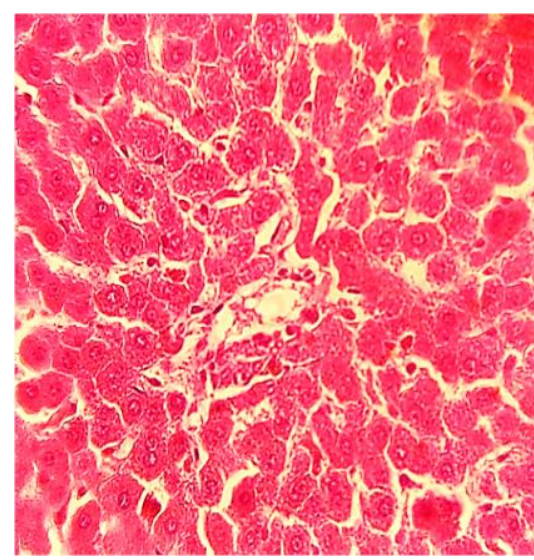

Show fat deposits in the hepatocytes and necrosis in alcoholic group (X-40).

(D)

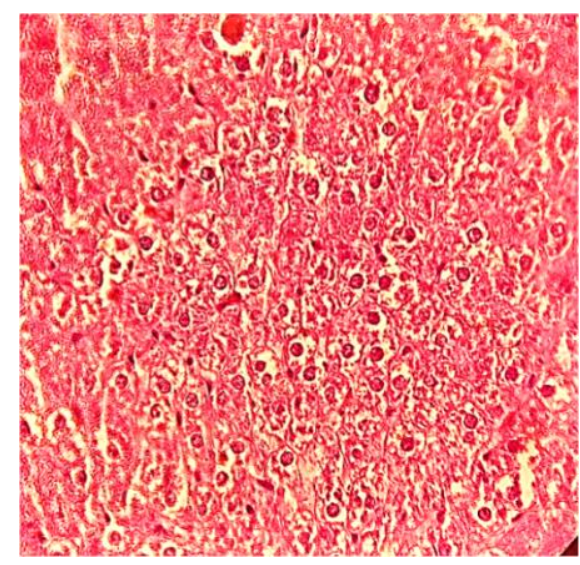

Show slight fat accumulation in liver cell in low dose cocoa treated group in comparison with alcoholic group (X-40).

(F)

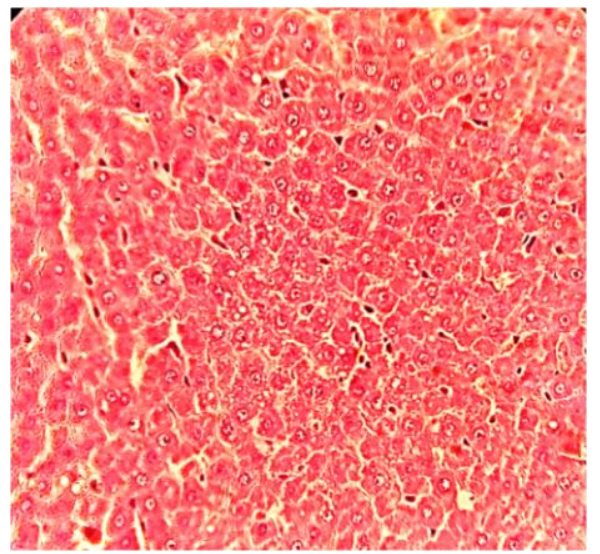

Show absence of necrosis and reduction in fat accumulation in liver cell after galaxy chocolate administration in comparison to alcoholic group (X-40).

efficiency of row cocoa and processed cocoa (galaxy chocolate) in the treatment of alcoholic fatty liver and compares them with the one of common drugs used to treat this situation (prednisolone).

The results of this study show that the groups received cocoa with high and low doses, galaxy chocolate and prednisolone have significant decrease in serum ALT enzyme when compare with positive control which receive alcohol ( $\mathrm{p}$-value $=0.000)$, Also there was a significant decrease in the ALT level after administration of prednisolone drug when compared with high cocoa dose group $(\mathrm{p}=0.017)$.

Also there was a significant decrease in the mean of plasma AST concentration when use cocoa $(0.67$ and $1.35 \mathrm{~g} / \mathrm{kg})$, galaxy chocolate and prednisolone compared to positive control with $\mathrm{p}$-value 
(p-value $=0.000)$. Also there was a significant decrease in the AST level after administration of cocoa $(1.35 \mathrm{~g} / \mathrm{kg})$ compared to group treated by low dose of cocoa $(0.67 \mathrm{~g} / \mathrm{kg})(\mathrm{p}=0.004)$. Galaxy chocolate significantly reduce the mean concentration of AST compared to low dose cocoa treated group $(\mathrm{p}=0.006)$.

The present study show a significant decrease in the mean concentration of plasma GGT after treatment with cocoa $(0.67$ and 1.35 $\mathrm{g} / \mathrm{kg}$ ), galaxy chocolate and prednisolone compared to the positive control ( $p$-value $=0.000$ ). All of these findings in agreement with previous reports by Arellanes and others whom stated that, the presence of N-Caffeoyldopamine inTheobroma cacao tree, This natural compound showed a protective effect due to its antioxidant activity by increasing superoxide dismutase and glutathionelevels in hepatic tissue and significantly lowering the liver-enzyme levels (AST, ALT and ALP) (Arellanes et al. 2016).

Concerning the lipid profile, this study found that a significant decrease in the mean concentrations of plasma total cholesterol and LDL when use cocoa $(0.67$ and $1.35 \mathrm{~g} / \mathrm{kg})$, galaxy chocolate and prednisolone compared to positive control with $\mathrm{p}$-value ( $\mathrm{p}$ value $=0.000$ ). Also there was significant decrease in the total cholesterol level after cocoa administration $(0.67 \mathrm{~g} / \mathrm{kg})$ compared to galaxy chocolate treated group $(\mathrm{p}=0.000)$. Group administrated prednisolone had significant reduction in LDL concentration compared to galaxy chocolate and low cocoa doses $(p=0.007,0.013)$ respectively. High dose of cocoa significantly decrease the LDL levels compared to group received low cocoa dose $(p=0.02)$.

The mean concentration of plasma triglyceride was decreased significantly and the level HDL had significant increase after administration of cocoa $(0.67$ and $1.35 \mathrm{~g} / \mathrm{kg})$, galaxy chocolate and prednisolone compared to positive control with $\mathrm{p}$-value ( $\mathrm{p}$ value $=0.000)$. Also there was a significant decreased in the triglyceride level after cocoa administration $(0.67 \mathrm{~g} / \mathrm{kg}$ and $1.35 \mathrm{~g} / \mathrm{kg})$ and galaxy chocolate compared to prednisolone treated group $(\mathrm{p}=0.000,0.000$ and 0.000$)$ respectively. Concretely, with previous findings by Mckim et al. (2002), they was found that the cocoa extract prevent liver injury by alcohol consumption due to the presence of flavonoids which a potent antioxidants and reverse the fat accumulation and inflammation and decrease the level of 4hydroxynonenal, a product of lipid peroxidation (Mckimet al. 2002). The review presented by Franco and others investigate that a methyl xanthines in cocoa act as phosphodiesterase inhibitors; thus they stimulate the lipolysis and triglyceride break down through the oxidation pathway (Franco et al. 2013).

Also the HDL was increased significantly in galaxy chocolate treated group compared to that treated with low, high cocoa doses and prednisolone drug ( $\mathrm{p}=0.000,0.000$ and 0.000$)$ respectively. Prednisolone show significant increased in HDL concentration in comparison with low cocoa treated group $(\mathrm{p}=0.002)$. This finding was supported by Franco and others, they found that the"theobromine from cacao consumption significantly increases plasma HDL cholesterol levels, and decreases LDL concentration in plasma, conferring cardiovascular protection and reducing the risk of coronary heart disease" (Franco et al. 2013).

There was a significant differences between groups administrated low and high cocoa doses $(\mathrm{p}=0.008)$. All of these finding in agreement with (Jia et al. 2010) whom stated that, Short-term cocoa consumption significantly reduced blood cholesterol, LDL; this due to the presence of high concentration of polyphenols (Jia et al. 2010).

The present study showed that, significant decrease in TG: HDL and LDL: HDL when use galaxy chocolate in comparison with Positive control $(\mathrm{P}-\mathrm{value}=0.000)$. The TG: HDL ratio was significantly decreased in galaxy chocolate treated group compared to cocoa low dose $(0.67 \mathrm{~g} / \mathrm{k}$ ( $\mathrm{p}$-value $=0.02)$. Also this ratio had significant decreased in high dose cocoa $(1.35 \mathrm{~g} / \mathrm{kg})$ and galaxy chocolate compared to prednisolone treated group ( $\mathrm{p}$-value $=0.046$, $0.005)$ respectively.

The results ofliver histology indicate that, the cocoa with high dose $(1.35 \mathrm{~g} / \mathrm{kg})$ and galaxy chocolate can efficiently reduce the fatty changes and necrosis due to alcohol toxicity compared to positive control and low cocoa dose $(0.67 \mathrm{~g} / \mathrm{kg})$.

\section{Conclusion}

The data of present study suggested that, the raw cocoa and processed chocolate decreases cholesterol, LDL and triglycerides moreover increase HDL, on the other hand decrease liver enzymes activity. Therefore it could possess hypolipidemia and treatment effect against alcohol induced cytotoxicity.

\section{References}

[1] Andujar I, Recio M.C, Giner R.M, Rios J.L., (2012). Cocoa polyphenols their potential benefits for human health. Department of Farmacologia, Facultat de farmacia, Universitat de Valencia,Oxidative Medicine and Cellular Longevity journal, 10.1155, p $1-23$.

[2] Arellanes JAM, Rebolledo GAG, Fischer MM, Le on-Díaz R. (2016). Medical plant extracts and natural compounds with a hepatoprotective effect against damage. Asian Pacific Journal of Tropical Medicine,Unidad de Investigaci'onM'edica en Farmacología, $9(12)$

$1141-$ 1149.https://doi.org/10.1016/j.apjtm.2016.10.010.

[3] Chiedozie OI, Paul CC and Ezeikel C.D., (2015). Functional Assessments and Histopathology of Hepatic and Renal Tissues of Wistar Rats Fed with Cocoa Containing Diets, Journal of Nutrition \& Food Sciences, p 1-6.

[4] Ding R, Tian K B, Lihuang L, Weihe C, Jiang Y, Wang TY, and Wana BJ. (2012). Herbal medicines for the prevention of alcoholic liver disease: A review, Journal of Ethnopharmacology, Institute of Chinese, Medical Sciences, University of Macau, Macao, PR China, Department of Gastroenterology, China (144): p 457-465.

[5] EASL Clinical Practical Guidelines: Management of Alcoholic Liver Disease, journal of hepatology, vol 57, p 399-420.

[6] Franco R, Astibia OA and Pinilla MA. (2013). Health Benefits of Methylxanthines in Cacao and Chocolate- a review, Nutrients journal,Department of Biochemistry and Molecular Biology, University of Barcelona, Spain(5) 4159-4173.

[7] Gramenzi A, Caputo F, Biselli M, Kuria F, Loggi E, Andreone M and Bernardi M. (2006). Review article: alcoholic liver disease pathophysiological aspects and risk factors, Alimentary pharmacology \& therapeutics, department of medicine, Italy, 24, p 11511161 .

[8] Jia L, Liu X, Yi Bai Y, Hua Li S, Sun K, He C, and Hui R. (2010). Short-term effect of cocoa product consumption on lipid profile: a meta-analysis of randomized controlled trials, Am J ClinNutr, (92):218-225.https://doi.org/10.3945/ajcn.2009.28202.

[9] Mandrekar P and Szabo G., (2009). Signalling pathways in alcoholinduced liver inflammation, , journal of hepatology, University of Massachusetts Medical School, Department of Medicine, 364 Plantation Street, Worcester, MA, USA, 50(6): p 1258-1266.

[10] McKim, SE, Konno A, Gabele E, Uesugi T, Froh M, Sies H, Thurman, RG, Arteel GE. (2002). Cocoa extract protects against early alcohol-induced liver injury in the rat. Archives of Biochemistry and Biophysics, (406): p 40-46.https://doi.org/10.1016/S00039861(02)00425-3.

[11] Scapagnini G, Davinelli S ,Di Renzo L, De Lorenzo A, Olarte HH, Micali G, Cicero FA and Gonzalez S. ( 2014). Cocoa Bioactive Compounds: Significance and Potential for the Maintenance of Skin Health, Department of Medicine and Health Sciences, University of Molise, Campobasso 86100, Italy. Nutrients journal,(6), p 3202-3213.

[12] Subhashini R, MahadevaRao US, Sumathi P and Gayathri G. (2010). A comparative phytochemical analysis of cocoa and green tea. indian journal of science and technology, p 188-192. 